\title{
PTPRZ1 regulates calmodulin phosphorylation and tumor progression in small-cell lung carcinoma
}

\author{
Hideki Makinoshima ${ }^{1,2}$, Genichiro Ishii ${ }^{1}$, Motohiro Kojima' ${ }^{1}$, Satoshi Fujii', Youichi Higuchi ${ }^{2,3}$, Takeshi Kuwata \\ and Atsushi Ochiai ${ }^{1,3^{*}}$
}

\begin{abstract}
Background: Small-cell lung carcinoma (SCLC) is a neuroendocrine tumor subtype and comprises approximately $15 \%$ of lung cancers. Because SCLC is still a disease with a poor prognosis and limited treatment options, there is an urgent need to develop targeted molecular agents for this disease.

Methods: We screened 20 cell lines from a variety of pathological phenotypes established from different organs by RT-PCR. Paraffin-embedded tissue from 252 primary tumors was examined for PTPRZ1 expression using immunohistochemistry. shRNA mediated PTPRZ1 down-regulation was used to study impact on tyrosine phosphorylation and in vivo tumor progression in SCLC cell lines.

Results: Here we show that PTPRZ1, a member of the protein tyrosine- phosphatase receptor (PTPR) family, is highly expressed in SCLC cell lines and specifically exists in human neuroendocrine tumor (NET) tissues. We also demonstrate that binding of the ligand of PTPRZ1, pleiotrophin (PTN), activates the PTN/PTPRZ1 signaling pathway to induce tyrosine phosphorylation of calmodulin (CaM) in SCLC cells, suggesting that PTPRZ1 is a regulator of tyrosine phosphorylation in SCLC cells. Furthermore, we found that PTPRZ1 actually has an important oncogenic role in tumor progression in the murine xenograft model.
\end{abstract}

Conclusion: PTPRZ1 was highly expressed in human NET tissues and PTPRZ1 is an oncogenic tyrosine phosphatase in SCLCs. These results imply that a new signaling pathway involving PTPRZ1 could be a feasible target for treatment of NETs.

Keywords: Small cell lung carcinoma (SCLC), Protein tyrosine phosphatase (PTP), Protein tyrosine phosphatase receptor Z1 (PTPRZ1), NETs (Neuroendocrine tumors), Pleiotrophin (PTN), Calmodulin (CaM)

\section{Background}

Neuroendocrine tumors (NETs) that includes small cell lung carcinomas (SCLC), large cell neuroendocrine carcinomas (LCNEC), pancreatic neuroendocrine tumors (PanNET), medullary thyroid carcinomas (MTC), pheochromocytomas, paragangliomas, and carcinoids [1-4]. As one of the most malignant NETs, SCLC comprises

\footnotetext{
* Correspondence: aochiai@east.ncc.go.jp

${ }^{1}$ Pathology Division, Research Center for Innovative Oncology, National Cancer Center Hospital East, 6-5-1 Kashiwanoha, Kashiwa, Chiba, 277-8577, Japan

${ }^{3}$ Laboratory of Cancer Biology, Department of Integrated Biosciences, Graduate School of Frontier Sciences, The University of Tokyo, Kashiwa, Chiba, Japan

Full list of author information is available at the end of the article
}

approximately $15 \%$ of lung cancer cases, and basic and clinical research efforts have translated little innovation in the treatment of this disease over the past 30 years [5]. Although SCLC appears to be effectively controlled with first line chemotherapy because of its relative high sensitivity to chemotherapy and radiotherapy, most patients ultimately relapse and salvage chemotherapy is considered [6]. To identify novel drug targets against SCLC, a greater understanding of the pathology of SCLC through molecular analysis is urgently needed.

Dissection of the signaling pathways that may be involved in the regulation of SCLC growth, for example via phosphorylation or dephosphorylation of critical proteins, may shed light on new approaches for tumor

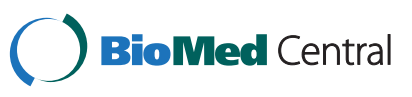


elimination. Protein tyrosine phosphorylation is tightly regulated by protein tyrosine-kinases (PTKs) and protein tyrosine-phosphatases (PTPs) [7,8]. PTPs play an important role in the inhibition and control of growth as tumor suppressors, since aberrant tyrosine phosphorylation is a characteristic feature of cancer cells [7-9]. Indeed, PTPs expressed as cell surface receptors (PTPRs) have been reported to be inactivated by genetic mutations in human cancer $[9,10]$. On the other hand, there is mounting evidence suggesting that several PTPRs also have oncogenic function [9].

PTPRZ1, as a member of the PTPR family, is a singlepass type I membrane protein with two cytoplasmic tyrosine phosphatase domains (D1 and D2), an alphacarbonic anhydrase domain (CA), chondroitin sulfate proteoglycans (CS-PGs) and a fibronectin type-III domain (FNIII) [11]. PTPRZ1 interacts with its ligand pleiotrophin (PTN), which is a secreted growth factor involved in angiogenesis and tumor growth [12,13]. Upon binding, PTN inactivates the phosphatase activity of PTPRZ1, which leads to an increased tyrosine phosphorylation status of important signaling molecules such as $\beta$-catenin, Fyn and RhoGAP [14-18]. With regard to cancer, PTPRZ1 expression was dramatically induced by genetic amplification caused by chronic oxidative stress and hypoxic stress through HIF-2 alpha [14,19] and several previous studies suggested that PTPRZ1 regulates cancer cell growth and cell migration [18,20-24].

In this paper, we found that PTPRZ1 is highly expressed in SCLC cell lines and specifically exists in human NET tissues. We hypothesized that PTPRZ1 functions to regulate tyrosine phosphorylation in SCLC cells and has an important role for SCLC tumor progression. To test this idea, we investigated the ability of PTPRZ1 to regulate tyrosine phosphorylation and tumor progression using SCLC cell lines.

\section{Methods}

\section{Cell cultures}

LN229 (glioblastoma, ATCC\#CRL-2611), U87MG (glioblastoma/astrocytoma, ATCC\#HTB-14), Hela (cervix ADCA, ATCC\#CCL-2), Caco2 (colorectal ADCA, ATCC\#HTB-37), DLD1 (colorectal ADCA, ATCC\#CCL221), HCT116 (colorectal ADCA, ATCC\#CCL-247), SW480 (colorectal ADCA, ATCC\#CCL-228), A549 (lung ADCA, ATCC\#CCL-185), LNCaP (prostate ADCA, ATCC\#CRL-1740), MCF7 (breast ADCA, ATCC\#HTB22), A431 (squamous cell carcinoma, ATCC\#CRL-1555), NCI-H69 (SCLC, ATCC\#HTB-119), NCI-H82 (SCLC, ATCC\#HTB-175), NCI-H345 (SCLC, ATCC\#HTB-180), NCI-H446 (SCLC, ATCC\#HTB-171), NCI-H510A (SCLC, ATCC\#HTB-184), NCI-H1436 (SCLC, ATCC\#CRL-5871), and NCI-H1930 (SCLC, ATCC\#CRL-5906) were originally purchased from ATCC and stocked in our Research
Center. TE1 (esophagus squamous cell carcinoma), TE3 (esophagus squamous cell carcinoma), TE4 (esophagus squamous cell carcinoma), TE5 (esophagus squamous cell carcinoma), and TE10 (esophagus squamous cell carcinoma) were gifts from Dr. Sasaki (National Cancer Center Research Institute). SBC-3 (SCLC, \#JCRB0818) was obtained from the JCRB and stocked in our Research Center. All cell lines were cultured in cell culture dishes (BD Biosciences) at $37^{\circ} \mathrm{C}$ and $5 \%$ carbon dioxide using RPMI 1640 (SIGMA), DMEM (SIGMA) supplemented with 10\% fetal bovine serum (FBS, Nichirei Bioscience), or HITES Medium [25,26] supplemented with penicillin/streptomycin (Invitrogen). For the PTN assay, $100 \mathrm{ng} / \mathrm{ml}$ of recombinant human pleiotrophin/PTN (R\&D Systems \#252-PL) was used.

\section{Human cancer samples}

Samples were obtained with informed consent from each individual, and the study was approved by the Ethics Committee of the National Cancer Center East Hospital. During the period from January 1992 to December 2010, a total of 252 patients who had primary tumors were treated at the National Cancer Center Hospital East, Chiba, Japan. All primary cancers with a pathologic diagnosis based on the classification schema of the WHO classification were reviewed, with 105 cases as adenocarcinoma (ADC), 61 as squamous cell carcinoma (SQCC) and 86 as neuroendocrine tumors (NETs). We used tissue microarray (TMA) to measure PTPRZ1 expression within lung tumors [27]. Each case in which more than $80 \%$ of the cancer cells reacted positively for an antibody to PTPRZ1 was recorded as positive.

\section{Antibodies}

Antibodies used included anti-PTPRZ1 (SIGMA \#015103) [28], anti-Phosphotyrosine, clone 4 G10 (Millipore \#05-321), anti-Calmodulin (Santa Cruz sc-5537, Millipore \#05-173, abcam ab45689), anti-phospho-Calmodulin (Santa Cruz Biotechnology sc-23760-R, Millipore \#09-295) and anti- $\beta$-tublin (Cell Signaling \#2146).

\section{Immunohistochemistry (IHC)}

All immunohistochemical (IHC) analyses were performed on paraffin-embedded tissues obtained from the primary tumor in the surgical specimen. For all IHC analyses the surgically resected specimens were fixed in $10 \%$ formalin and embedded in paraffin for routine pathological examination. We prepared and used 5- $\mu \mathrm{m}$ thick paraffin sections cut from a paraffin block containing histological findings that were representative of the tumor. The procedure for IHC was previously described $[27,28]$. Antigen retrieval was performed in citrate buffer solution ( $\mathrm{pH}$ 6.0). Endogenous peroxidase was blocked with $0.3 \% \mathrm{H}_{2} \mathrm{O}_{2}$ in methanol for $15 \mathrm{~min}$ and all slides 
were heated to $95^{\circ} \mathrm{C}$ by exposure to microwave irradiation for $20 \mathrm{~min}$ and then cooled at room temperature (RT). Slides were washed in PBS and after a $1 \mathrm{~h}$ incubation at RT with the primary antibodies, the slides were incubated for 30 min with a labeled polymer EnVision $\mathrm{TM}+$, Peroxidase-conjugated anti-Mouse or Rabbit (Dako, Tokyo, Japan). The chromogen used was $2 \% 3$, $3^{\prime}$-diaminobenzidine (DAB) in $50 \mathrm{mM}$ Tris-buffer (pH 7.6) containing $0.3 \%$ hydrogen.

\section{RNA isolation and real-time RT-PCR}

Cells were washed with PBS and total RNA from the cell lines was isolated with TRIzol Reagent (Invitrogen). Complementary DNA (cDNA) was synthesized using the PrimeScript ${ }^{\circledR}$ RT reagent Kit (TaKaRa, Japan). Real-time RT-PCR was carried out with specific primers and a Smart Cycler (Cepheid, Sunnyvale, CA, USA). Real-time fluorescence monitoring of the PCR products was performed with SYBR Green I fluorescent dye (TaKaRa). The levels of expression of specific genes are reported as ratios to the level of expression of GAPDH in the same master reaction. Synthesized primers were purchased from TaKaRa Bio with Primer Set ID given as PTPRZ1, $3^{\prime}$ (HA082543). GAPDH was used for normalization as control and the relative quantitation value compared to the calibrator for that target is expressed as $2^{-(\mathrm{Ct}-\mathrm{Cc})}$.

\section{Western blot}

Western blotting was performed as described [29]. After lentivirus infection with the vector for $\operatorname{sh} L U C$ or shPTPRZ1, total cell lysate was prepared from cells cultured in complete medium. Primary antibodies were used at 1:1000 dilution and $\beta$-tubulin was used as loading control.

\section{Expression of short hairpin RNA (shRNA)}

Plasmid construction was carried out with Gateway system (Invitrogen) according to the manufacturer's instructions. Cloning vectors were pDNOR221 (Invitrogen) and pENTR/U6 (Invitrogen). The lentiviruses were produced using 293FT cells (Invitrogen) transfected with pCAGHIVgp, pCMV-VSV-G-RSV-Rev, and a lentivirus vector based on CSII-CMV-RfA-IRES2-Venus (Dr. Miyoshi, RIKEN BioResource Center) expressing shRNA with the sequence described below. Transfection was achieved using Lipofectamine 2000 reagent (Invitrogen) according to the manufacturer's instructions. Lentivirus-containing medium was filtered through a $0.45 \mu \mathrm{m}$ filter and used for transduction of target cells. The sequences and plasmid names were; shLUC: GTGCGCTGCTGGTGCCAAC (pGL3, firefly luciferase), shPTPRZ1_1: GCCTATAAATTGTGAGAGCTT (pHMA017), shPTPRZ1_2: GCTGCTTTAGATCCATTCATA (pHMA019), and shPTPRZ1_3: GGATGGCAAACTGACTGAT (pHMA022).

\section{Flow cytometry}

Cells were incubated with anti-PTPRZ1 antibody (SIGMA) and excess antibody was removed by washing with PBS containing 2\% FBS. Polyclonal goat antirabbit immunoglobulin conjugated to Phycoerythrin (PE) (Jackson) was added as a secondary antibody. The cells were then washed with PBS and flow cytometric analysis was performed using a FACSCalibur and FACSAria (BD Biosciences).

\section{Animal studies}

All of experimental SCID mice were handled in accordance with institutional guidelines established by the Animal Care Committee of the National Cancer Center East Hospital. H69 and H1930 SCLC cells expressing shRNA were injected into the subcutaneous tissue of SCID mice (7-8 weeks of age, CLEA, Tokyo, Japan). Tumor volume was calculated as the product of a scaling factor of 0.52 and the tumor length, width, and height were measured every week. For IHC analysis, organs were obtained from mice at 5 or 8 weeks after injection and fixed in $10 \%$ formalin.

\section{Statistical methods}

Standard Student's $t$-test was used to determine the significance between non-targeting control and shPTPRZ1 experiments. Statistical correlation was carried out using $X^{2}$ test for independence $(2 \times 2$ contingency table $)$. $P<0.05$ was considered statistically significant.

\section{Results}

\section{PTPRZ1 is highly expressed in SCLC cell lines}

To assess mRNA expression of PTPRZ1 comprehensively in human cancers, we screened 20 cell lines from a variety of pathological phenotypes established from different organs by RT-PCR. We observed that two SCLC cell lines at the first screening, NCI-H69 (H69) and NCI-H1930 (H1930), expressed PTPRZ1 mRNA at significantly higher levels than other cell lines (Figure 1A). To confirm the specificity of PTPRZ1 expression in SCLC cells, we measured PTPRZ1 protein levels by Western blotting (Figure 1B). The human PTPRZ1 gene encodes a core protein consisting of 2315 amino acids (NCBI Reference Sequence: NP_002842) with a predicted molecular weight (M.W.) of $400 \mathrm{kDa}$, [30]. Indeed, we detected a specific band of PTPRZ1 protein at approximately $400 \mathrm{kDa}$ by WB, only within SCLC cell lines expressing PTPRZ1 mRNA at high levels (Figure 1B).

\section{PTPRZ1 is specifically expressed in human NET tissues}

To determine globally which human tumor tissues expressed PTPRZ1, we analyzed immunohistochemical (IHC) evaluations of a variety of tumors including 105 


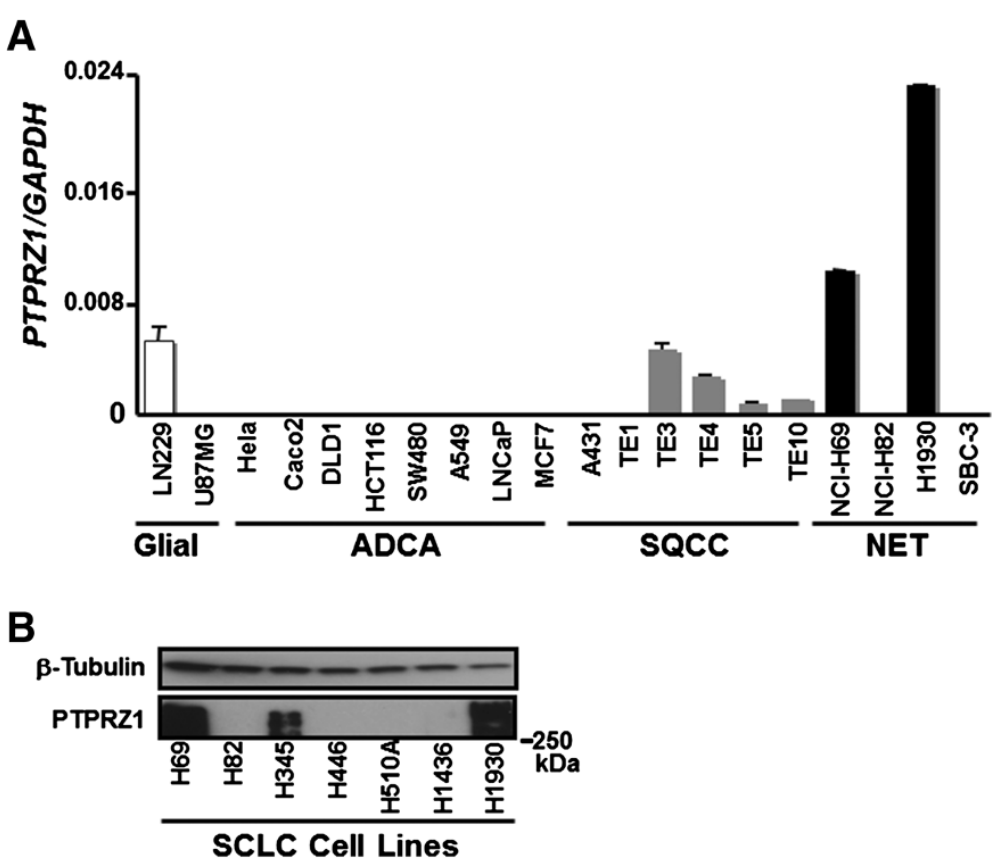

Figure 1 Gene expression of PTPRZ1 among the different cancer cell lines. Glial, adenocarcinoma (ADCA), squamous cell carcinoma (SQCC) and neuroendocrine tumor (NET) cell lines were screened for PTPRZ1 mRNA levels (normalized to GAPDH), with standard deviation error bars shown (A). PTPRZ1 was expressed in SCLC cell lines at protein level (B).

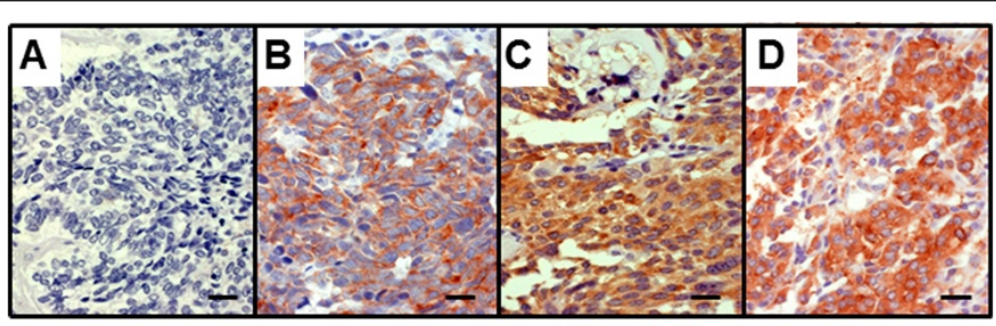

E

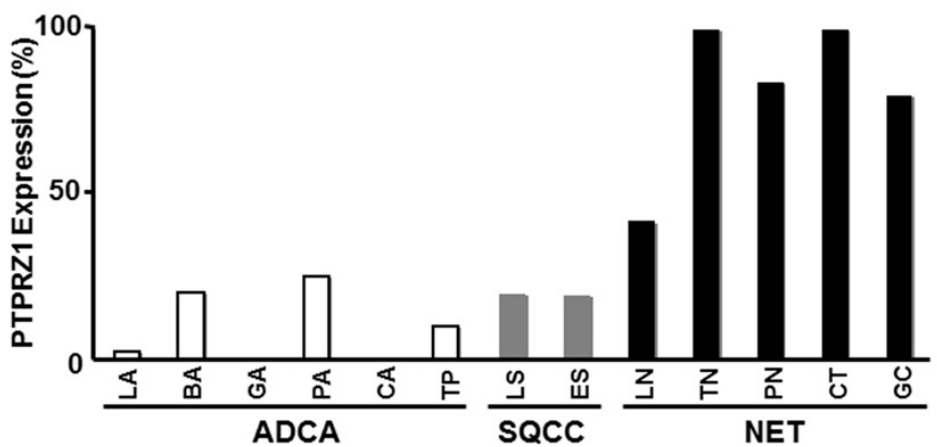

Figure 2 PTPRZ1 was expressed in human NETs. A-D, Representative microscopic images for PTPRZ1-negative SCLC (A), PTPRZ1-positive SCLC (B), Thyroid Medullary carcinoma (TMC) (C) and Pancreatic endocrine tumor (PanNET) (D). Scale bars are $20 \mu \mathrm{m}$ (Magnification X40). E, The percentage of PTPRZ1 expression in human tumor tissues was measured in ADCA, SQCC, and NET pathological types including lung ADCA (LA), breast ADCA (BA), gastric ADCA (GA), pancreatic ADCA (PA), colon ADCA (CA), Thyroid ADCA (TA), lung SQCC (LS), esophagous SQCC (ES),lung NET (LN), thyroid NET (TN), pancreatic NET (PN), chromaffin-cell NET (CT), and gastrointestinal carcinoid (GC). 
cases of adenocarcinoma (ADCA), 61 cases squamous cell carcinoma (SQCC) and 86 cases NET. In non-tumor tissues, we specifically observed PTPRZ1 expression in the neural cells and endocrine cells such as peripheral nerves, pancreatic islets and adrenal chromaffin cells. Representative IHC evaluations of PTPRZ1-positivity (PTPRZ1+) with anti-PTPRZ1 antibody in a variety of NETs are shown in Figure 2, for PTPRZ1-negative (PTPRZ1-) SCLC (A), PTPRZ1+ SCLC (B), MTC (C), and PanNET (D). PTPRZ1 was mainly localized in the cell membrane as well as the cytosol. We found that PTPRZ1 was detected at high frequency and intensity in a variety of human NETs including $60 \%$ of SCLCs (Figure 2E, Table 1). PTPRZ1 was expressed at much higher levels in NETs (79\%) than in ADCA (9\%) and SQCC (20\%) (Figure 2E).

\section{RNAi knockdown of PTPRZ1 in SCLC cell lines}

To characterize further the function of PTPRZ1 in SCLC cells, we employed a genetic approach to repress PTPRZ1 expression using by RNA interference (RNAi). For potential off-target shRNA effects, three different sequences of shRNA directed against PTPRZ1 (shZ1\#1, $\# 2$ and \#3) and a nontargeting shRNA ( $\operatorname{sh} L U C$ ) were constructed. While the introduction of the first construct shZ1\#1 in SCLC cells did not appear to downregulate PTPRZ1 mRNA levels as compared to control shLUC when measured by quantitative RT-PCR, significant reduction in mRNA expression of $75 \%$ using shZ1\#2 and $60 \%$ using shZ1\#3 could be observed in the expression of PTPRZ1 in the SCLC cell lines H69 and

Table 1 The IHC analysis of PTPRZ1 expression in human tumor tissues

\begin{tabular}{lccc}
\hline Tumors & PTPRZ1- & PTPRZ1+ & \% \\
\hline Adenocarcinoma (ADCA) & 44 & 1 & $\mathbf{9}$ \\
Lung ADCA & 8 & 2 & 20 \\
Breast ductal ADCA & 10 & 0 & 0 \\
Gastric ADCA & 15 & 5 & 25 \\
Pancreatic ductal ADCA & 10 & 0 & 0 \\
Colon ADCA & 9 & 1 & 10 \\
Thyroid papillary carcinoma & & & $\mathbf{2 0}$ \\
Squamous cell carcinoma (SQCC) & 41 & 10 & 20 \\
Lung SQCC & 8 & 2 & 20 \\
Esophagus SQCC & & & $\mathbf{7 9}$ \\
Neuroendocrine tumor (NET) & 11 & 8 & 42 \\
Lung NET & 0 & 16 & 100 \\
Medullary thyroid carcinoma & 5 & 26 & 84 \\
Pancreatic NET & 0 & 10 & 100 \\
Chromaffin cell tumor & 8 & 2 & 80 \\
Gastointestinal carcinoid & & & 2 \\
\hline
\end{tabular}

H1930 (Figure 3A). WB analyses also revealed significant decreases in PTPRZ1 protein expression upon introduction of shZ1\#2 and \#3, as compared to a control vector, in H69 and H1930 under normal culture conditions (Figure 3B). To measure cell surface PTPRZ1 levels in shZ1-transduced SCLC cells, we used flow cytometry (FACS). FACS analysis of shLUC-SCLC cells and shZ1SCLC cells also revealed significant reduction of PTPRZ1 expression on SCLC cellular surface from 29\% to $6-7 \%$ in $\mathrm{H} 69$ cells and $37 \%$ to $9-12 \%$ in $\mathrm{H} 1930$ cells (Figure 3C).

\section{PTN induced calmodulin tyrosine phosphorylation in SCLC cells}

Although our findings demonstrated that PTPRZ1 was specifically up-regulated in SCLC cells, no studies to date have suggested a functional role for PTPRZ1 in SCLC cells. As PTPRZ1 has been linked to protein tyrosine phosphatase activity, we first assessed the ability of PTPRZ1 to regulate tyrosine phosphorylation in the response to the ligand of PTPRZ1, PTN. PTN binding to the extracellular portion of PTPRZ1 brings two molecules into close proximity and consequently the phosphatase domains dimerize in a head-to-toe arrangement with the D2 domain of one molecule blocking the active site (D1) of the second molecule, leading to suppression of phosphatase activity [31,32]. To identify molecular targets regulated by PTPRZ1 in response to PTN, we assessed tyrosine-phosphorylated proteins using an antiphosphotyrosine antibody by WB. Interestingly, we detected two specific bands that migrated just above and below $15 \mathrm{kDa}$ within $30-60 \mathrm{~min}$ after PTN addition to SCLC cells (Figure 4A). Although it appears that those bands could be detected at low levels in the absence of PTN, the addition of PTN significantly induced phosphorylation that peaked at $1 \mathrm{~h}$. Since calmodulins (CaM) are highly abundant, $17 \mathrm{kDa}$ proteins in the mammalian brain, nervous and endocrine systems and directly interact with the intracellular domain of PTPR members $[33,34]$, we hypothesized that PTPRZ1 may normally dephosphorylate the phosphorylated tyrosine residue at Tyr99 of CaM. To test this idea, we assessed the phosphorylation of CaM using an anti- phospho-Tyr99-CaM $(\mathrm{p}-\mathrm{CaM}) \mathrm{Ab}$ and determined that the upper band could indeed be identified as CaM (Figure 4B).

\section{PTPRZ1 is required for the tyrosine phosphorylation of CaM induced by PTN}

To verify that the addition of PTN facilitated CaM phosphorylation specifically through its receptor PTPRZ1, we utilized the H69 and H1930 cell lines in which shZ1 was used to knock down PTPRZ1 expression. Although PTN induced tyrosine phosphorylation of CaM in $\mathrm{H} 69$ cells that expressed the control shLUC, the ablation of 

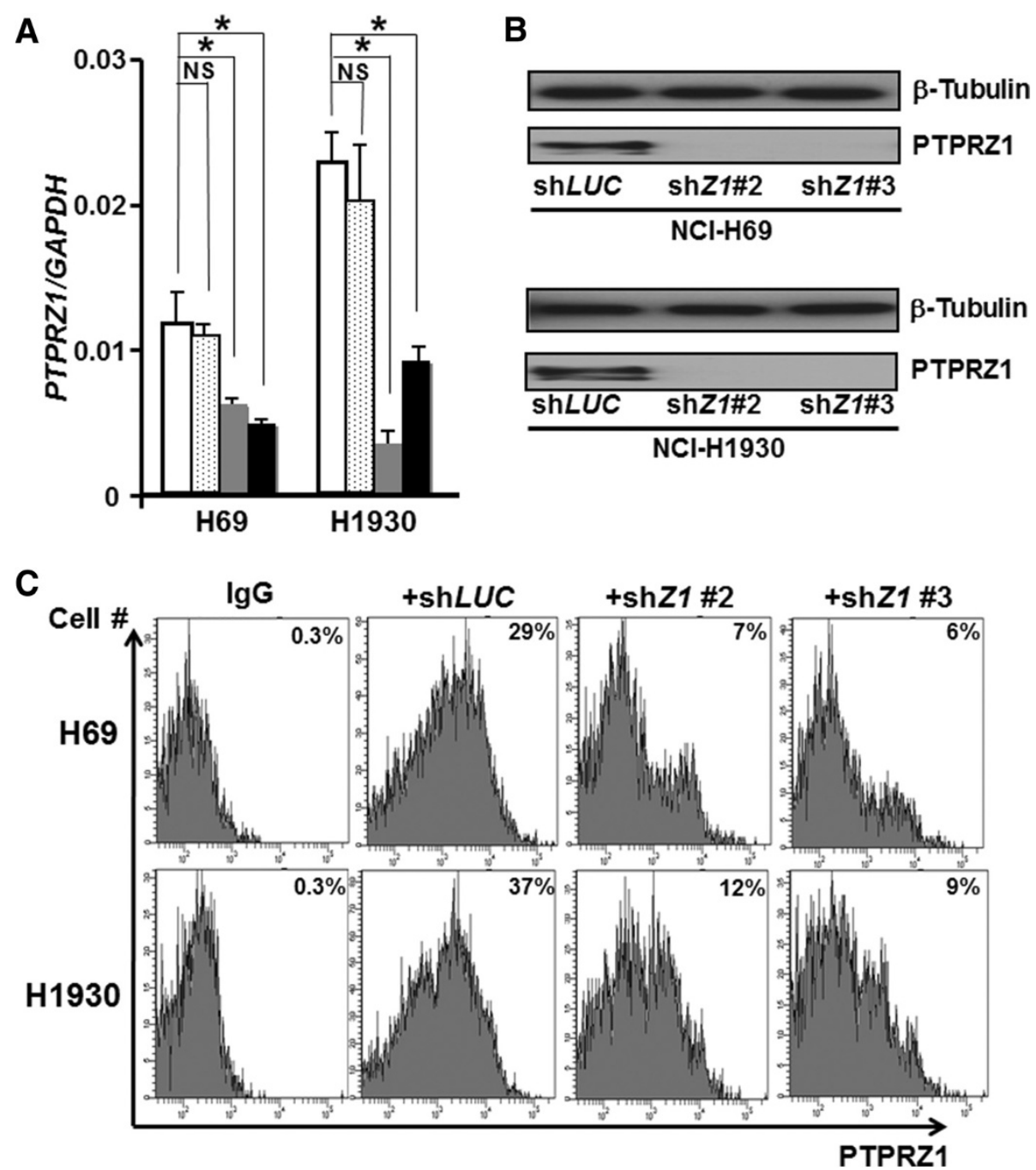

Figure 3 PTPRZ1 expression was downregulated by shRNA in SCLC cell lines. A, shRNA targeting PTPRZ1 (shZ1) successfully knock-downed PTPRZ1 mRNA by up to 75\% in the SCLC cell lines, $\mathrm{H} 69$ and H1930, as compared to negative control construct expressing shLUC. White bars = shLUC, dotted bars $=$ shZ1\#1, gray bars $=$ shZ1\#2, black bars $=$ shZ1\#3. Error bars represent SD. Asterisk denotes $P<0.05$ using Student's $t$ test, while NS denotes non-significant change. B, PTPRZ1 downregulation in $\mathrm{H} 69$ and $\mathrm{H} 1930$ was confirmed by Western blot, using $\beta$-tubulin as control for protein levels. C, FACS analysis of surface PTPRZ1 protein expression on H69 and H1930 cells, with shZ1 down-regulating PTPRZ1 expression levels.

PTPRZ impaired PTN-induced CaM tyrosine phosphorylation in cells that expressed either of the two shZ1 constructs (Figure 4C). We confirm that PTPRZ1 was indispensable for the PTN-induced p-CaM in H1930 cells (Figure 4D).

As the serum levels of PTN were elevated in most SCLC patients in comparison to healthy controls [12], we thought PTPRZ1 expression might be correlate with the expression of phosphorylated CaM. To assess whether PTPRZ1-CaM regulation also occurred in vivo in human tissue, we stained for $\mathrm{CaM}$ and $\mathrm{p}$-CaM. Indeed we found that the expression of PTPRZ1 and p-CaM was statistically correlated in human lung NET tissues (Figure 4E). These data thus demonstrate that ablation of PTPRZ1 prevents PTN-stimulated tyrosine phosphorylation of CaM in PTN-stimulated SCLC cells; the data indicate that endogenous PTPRZ1 is required for PTNstimulating tyrosine phosphorylation of CaM in SCLC cells.

\section{PTPRZ1 regulates tumor progression of SCLC in xenograft model}

Many PTPRs play an important role as tumor suppressors [9], yet PTPRZ1 has a role in cell migration and tumor growth in vivo in glioma studies [20]. To determine whether overexpressed PTPRZ1 acts as a tumor suppressor or tumor promoter in human NETs, we used the severe combined immunodeficiency (SCID) murine 


\section{A}

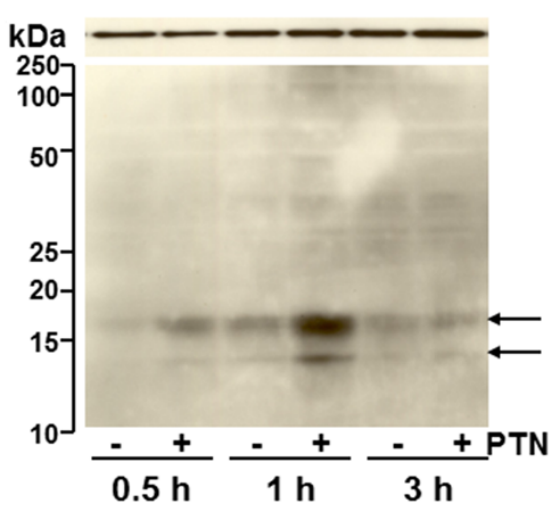

C

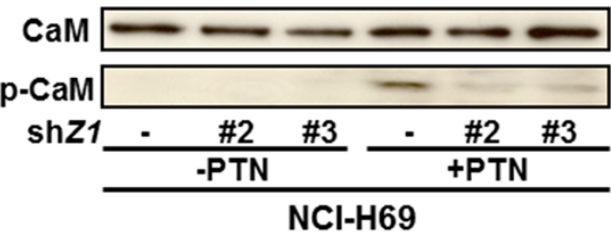

B

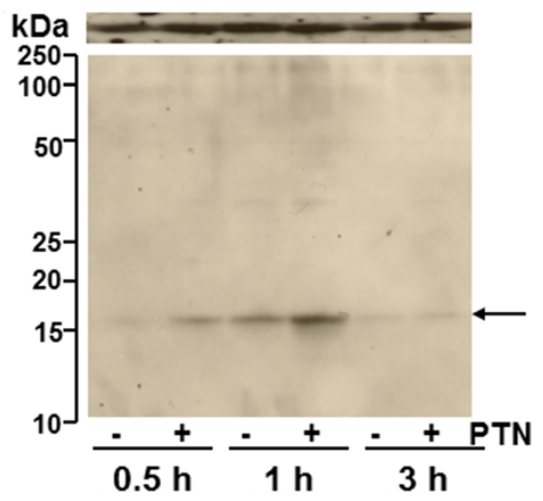

D

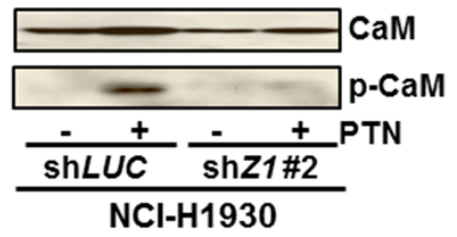

E
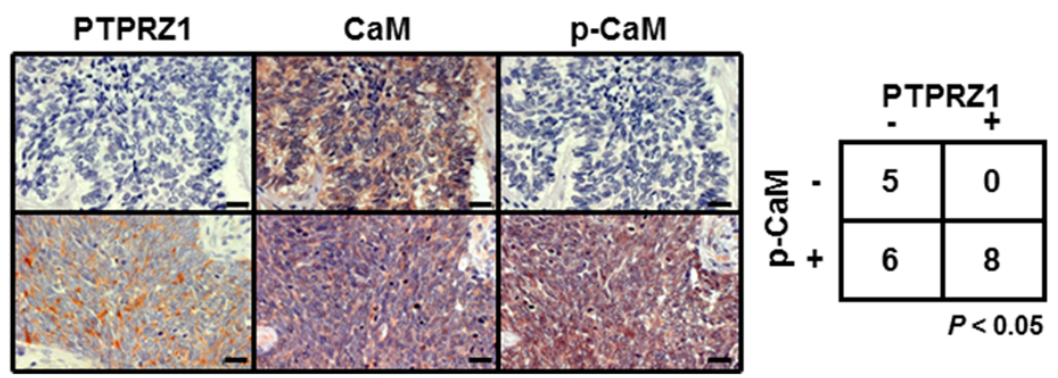

Figure 4 PTPRZ1 regulates tyrosine phosphorylation in SCLC cells. A, Tyrosine phosphorylated proteins in H69 cells were detected by Western blot with anti-pTyr antibody (4 G10) with $\beta$-catenin as loading control on top. B, Tyrosine phosphorylated CaM was detected by Western blot with anti-phosphorylated CaM, with total CaM on top as loading control in H69 cells. C and D, shZ1s block PTN-stimulated tyrosine phosphorylation of CaM in H69 (C) and H1930 (D). PTPRZ1 promotes the phosphorylation of calmodulin in response to PTN. E, Correlated expression of PTPRZ1 and tyrosine phosphphorylated CaM (pCaM) in human lung NET tissues. Representative negative (-) or positive (+) images were shown. Scale bars were $20 \mu \mathrm{m}$ (Magnification X40). $P<0.05$ was considered statistically significant.

xenograft model subcutaneously transplanted with human SCLC cells. $2 \times 10^{6} \mathrm{H} 69$ cells expressing either $\operatorname{sh} L U C(\mathrm{H} 69+\operatorname{sh} L U C)$ as a control or $\operatorname{sh} Z 1 \# 2(\mathrm{H} 69+$ shZ1\#2) were subcutaneously transplanted into the flanks of SCID mice $(\mathrm{n}=7)$ and tumor size was measured over time. In this mouse model, $\mathrm{H} 69+\mathrm{sh} L U C$ cells started to grow exponentially at 7 days posttransplant and progressively form tumor masses for 5 weeks (Figure $5 \mathrm{~A}$ ). In contrast, the $\mathrm{H} 69+\operatorname{sh} Z 1 \# 2$ cells were impaired for tumor formation until 3 weeks posttransplant such that tumors were barely recognized under the skin and were about 3-fold smaller than those in $\mathrm{H} 69+\mathrm{sh} L U C$ cells (Figure $5 \mathrm{~A}$ and B). Gross examination of $\mathrm{H} 69+\operatorname{sh} Z 1 \# 2$ tumors revealed a dramatic loss of SCLC pathology in the tumor (Figure 5B). To exclude the possibility of off-target effects of shZ2\#2, we subcutaneously transplanted H69 cells expressing either $\operatorname{sh} L U C(\mathrm{H} 69+\operatorname{sh} L U C)$ as a control or shZ1\#3 (H69+ shZ1\#3) into the flanks of SCID mice $(n=7)$ and we obtained similar results (Figure $5 \mathrm{C}$ ). In another SCLC cell line, H1930, the reduction of PTPRZ1 expression decreased the rate of tumor formation under the skin in SCID mice as compared to the cells expressing the shLUC control (Figure 5D). These results provide proof that PTPRZ1 regulates tumor growth in vivo and has an oncogenic function in NET progression.

\section{Discussion}

Here we demonstrate that PTPRZ1 specifically exists in human NET tissues and PTPRZ1 has an important oncogenic role in the tumor progression of SCLC in the murine xenograft model. We also found that PTPRZ1 


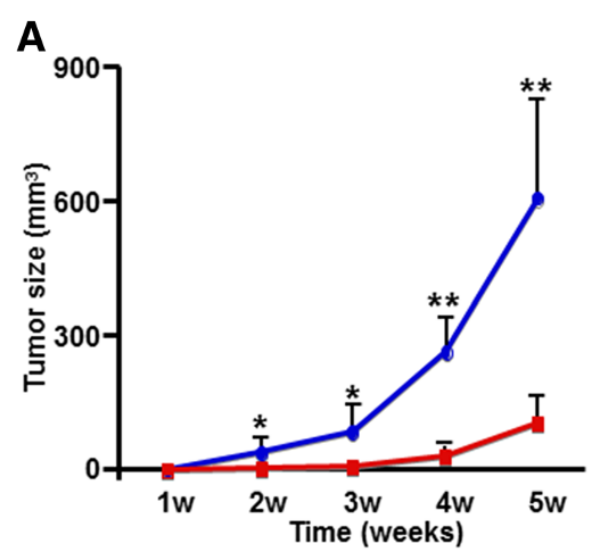

B
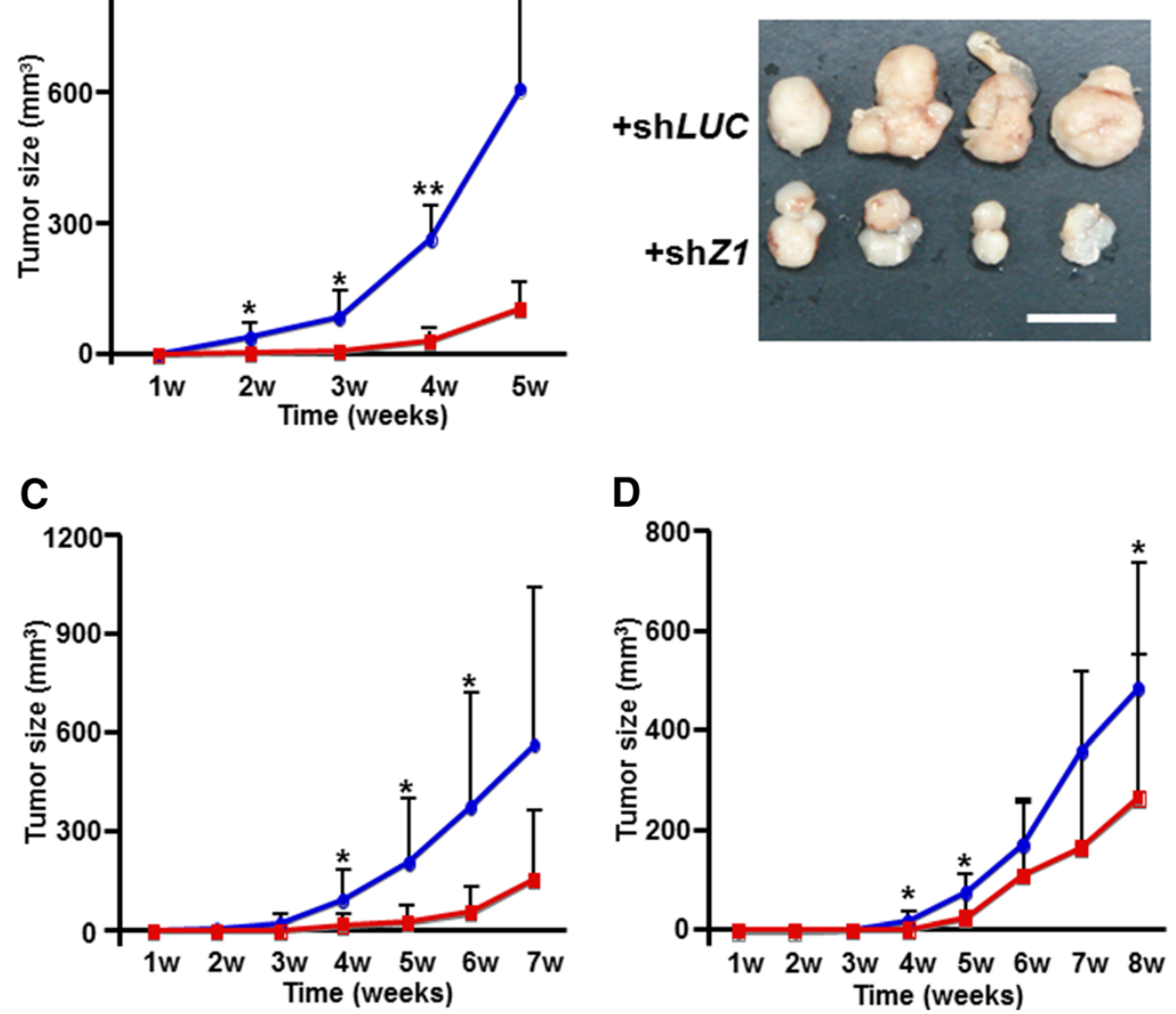

Figure 5 PTPRZ1 regulates tumor progression in a SCLC xenograft mouse model. A and B, in vivo growth of SCLC NCI-H69 expressing shLUC (blue diamonds) and shZ1\#2 (red squares) in SCID mice. The loss of PTPRZ1 in cells expressing shZ1\#2 inhibited tumor growth as compared to cells expressing the control shLUC. Tumor size $\left(\mathrm{mm}^{3}\right)$ over period (A) and gross tumor pathology 5 weeks post-transplant are shown (B). Scale bar indicates $10 \mathrm{~mm}$. C and D, Deficient in vivo growth of tumors was confirmed using H69 cells expressing shZ1\#3 (C) and H1930 cells expressing shZ1\#2. Control SCLC cells with shLUC are shown as blue diamonds while cells with shZ1 are shown as red squares. Error bars are SD. Asterisk, $P<0.05$; double asterisk, $P<0.01$; both using Student's $t$ test.

regulates the tyrosine phosphorylation of $\mathrm{CaM}$ in the response to PTN in SCLC cells. Our results indicate that the putative tumor suppressor family PTPR can support tumor progression and is required for the tyrosine phosphorylation of CaM. This study supports the idea that a new signaling pathway involving PTPRZ1 could be a feasible target for treatment of cancers. The combination of our cellular and xenograft model findings advocates for the future preclinical testing of antibody therapy or small molecule inhibitors of PTPRZ1 for the treatment of NETs and SCLC.

The linkage between oncogenic PTPRZ1 function and CaM phosphorylation is still unclear. Perez-Pinera and colleagues demonstrated that phosphorylation of Anaplastic lymphoma kinase (ALK) in PTN-stimulated cells is mediated through the PTN/PTPRZ1 signaling pathway [35], indicating that ALK might phosphorylate $\mathrm{CaM}$. Further experiments are needed to address the possibility of PTN mediating its effects via ALK [35] in
SCLC cells, the effects of PTN deletion on tumor growth, and the mechanism of PTN/PTPRZ1 autocrine regulation in NET cells. CaM can bind up to four calcium ions, and can undergo post-translational modifications such as phosphorylation, acetylation, methylation and proteolytic cleavage, each of which can potentially modulate its actions [34]. A prior biochemical study showed that tyrosine phosphorylation increased the association of CaM with nitric oxide synthase (NOS) [36]. Because nitric oxide (NO) and NOS are ubiquitous in malignant tumors and known to exert pro-tumor effects $[37,38]$, PTPRZ1 may regulate NO production in SCLC cells by changing the tyrosine phosphorylation status of CaM. Tumor cell-derived NO promotes tumor progression by induction of tumor-cell invasion, proliferation and the expression of angiogenic factors [37,38]. Indeed a recent research article demonstrated that glioma stem cell proliferation and tumor growth are promoted by iNOS [39]. 
With regards to another aspect of its oncogenic role, PTPRZ1 has a huge extracellular domain consisting of a alpha-carbonic anhydrase domain (CA), chondroitin sulfate proteoglycans (CS-PGs), and a fibronectin type-III domain (FNIII). PTPRZ1 expression is dramatically induced by hypoxic stress through HIF-2 $\alpha$ [19], suggesting that PTPRZ1 may have an important role under hypoxic conditions. Recently, Jeong's research group reported that CA was dramatically up-regulated in human SCLC tissues by proteomic analysis [40]. A possible speculation is that the CA domain of PTPRZ1 could have an important function for tumor progression of SCLC and further studies will be required to address this issue.

\section{Conclusions}

We found that PTPRZ1 has an important oncogenic role in tumor progression in the murine xenograft model of SCLCs. Moreover we demonstrate that the binding of PTPRZ1 to its ligand PTN inactivates phosphatase activity, resulting in tyrosine phosphorylation of $\mathrm{CaM}$ in human tumors. These results indicate that a new signaling pathway involving PTPRZ1 could be a feasible target for treatment of NETs.

\section{Abbreviations \\ SCLC: Small cell lung carcinoma; PTP: Protein tyrosine phosphatase; PTPRZ1: Protein tyrosine phosphatase receptor Z1; NETs: Neuroendocrine tumors; PTN: Pleiotrophin; CaM: Calmodulin; shRNA: Small Hairpin RNA.}

\section{Competing interests}

The authors declare that they have no competing interests.

\section{Authors' contributions}

Histological diagnostics for pathological human tissues were carried out by Gl, MK, SF, TK and AO. AO conceived the study. All experiments were optimized and performed by HM. Manuscript were written by $\mathrm{HM}$ and revised by $\mathrm{YH}, \mathrm{Gl}$ and $\mathrm{AO}$. All authors have read and approved this manuscript.

\section{Acknowledgements \\ We thank all Dr. Junichi Nitadori, Dr. Nao Atsumi, Ms. Hashimoto and Mr. Yanagi for outstanding technical supports and all of the Ochiai Lab members for support and discussions. We thank Dr. Phillip Wong for carefully reading the manuscript and providing critical comments. This work was supported by the Foundation for the Promotion of Cancer Research, 3rd-Term Comprehensive 10-Year Strategy for Cancer Control (AO, grant number: Section\#2). This study was also supported by the KAKENHI Grant-in-Aid for Young Scientists (B) from the Ministry of Education, Culture, Sports, Science and Technology of Japan (No. 24700990)}

\section{Author details}

${ }^{1}$ Pathology Division, Research Center for Innovative Oncology, National Cancer Center Hospital East, 6-5-1 Kashiwanoha, Kashiwa, Chiba, 277-8577, Japan. ${ }^{2}$ Research Resident, Foundation for Promotion of Cancer Research, Chuuou-ku 5-1-1 Tsukiji, Tokyo 104-0045, Japan. ${ }^{3}$ Laboratory of Cancer Biology, Department of Integrated Biosciences, Graduate School of Frontier Sciences, The University of Tokyo, Kashiwa, Chiba, Japan.

Received: 17 July 2012 Accepted: 15 November 2012 Published: 21 November 2012

\section{References}

1. Chen H, Sippel RS, O'Dorisio MS, Vinik Al, Lloyd RV, Pacak K: The North American Neuroendocrine Tumor Society consensus guideline for the diagnosis and management of neuroendocrine tumors: pheochromocytoma, paraganglioma, and medullary thyroid cancer. Pancreas 2010, 39(6):775-783.

2. Klimstra DS, Modlin IR, Coppola D, Lloyd RV, Suster S: The pathologic classification of neuroendocrine tumors: a review of nomenclature, grading, and staging systems. Pancreas 2010, 39(6):707-712.

3. Phan AT, Oberg K, Choi J, Harrison LH Jr, Hassan MM, Strosberg JR, Krenning EP, Kocha W, Woltering EA, Maples WJ: NANETS consensus guideline for the diagnosis and management of neuroendocrine tumors: well-differentiated neuroendocrine tumors of the thorax (includes lung and thymus). Pancreas 2010, 39(6):784-798.

4. Kulke MH, Anthony LB, Bushnell DL, de Herder WW, Goldsmith SJ, Klimstra DS, Marx SJ, Pasieka JL, Pommier RF, Yao JC, et al: NANETS treatment guidelines: well-differentiated neuroendocrine tumors of the stomach and pancreas. Pancreas 2010, 39(6):735-752.

5. William WN Jr, Glisson BS: Novel strategies for the treatment of small-cell lung carcinoma. Nat Rev Clin Oncol 2011, 8(10):611-619.

6. Kim YH, Mishima M: Second-line chemotherapy for small-cell lung cancer (SCLC). Cancer Treat Rev 2011, 37(2):143-150.

7. Stoker AW: Protein tyrosine phosphatases and signalling. J Endocrinol 2005, 185(1):19-33.

8. Tonks NK: Protein tyrosine phosphatases: from genes, to function, to disease. Nat Rev Mol Cell Biol 2006, 7(11):833-846.

9. Julien SG, Dube N, Hardy S, Tremblay ML: Inside the human cancer tyrosine phosphatome. Nat Rev Cancer 2011, 11(1):35-49.

10. Wang Z, Shen D, Parsons DW, Bardelli A, Sager J, Szabo S, Ptak J, Silliman N, Peters BA, van der Heijden MS, et al: Mutational analysis of the tyrosine phosphatome in colorectal cancers. Science 2004, 304(5674):1164-1166.

11. Shitara K, Yamada H, Watanabe K, Shimonaka M, Yamaguchi Y: Brain-specific receptor-type protein-tyrosine phosphatase RPTP beta is a chondroitin sulfate proteoglycan in vivo. J Biol Chem 1994 269(31):20189-20193.

12. Jager R, List B, Knabbe C, Souttou B, Raulais D, Zeiler T, Wellstein A, Aigner A, Neubauer A, Zugmaier G: Serum levels of the angiogenic factor pleiotrophin in relation to disease stage in lung cancer patients. $\mathrm{Br} \mathrm{J}$ Cancer 2002, 86(6):858-863.

13. Kadomatsu K, Muramatsu T: Midkine and pleiotrophin in neural development and cancer. Cancer Lett 2004, 204(2):127-143.

14. Liu YT, Shang D, Akatsuka S, Ohara H, Dutta KK, Mizushima K, Naito Y, Yoshikawa T, Izumiya M, Abe $K$, et al: Chronic oxidative stress causes amplification and overexpression of ptprz1 protein tyrosine phosphatase to activate beta-catenin pathway. Am J Pathol 2007, 171(6):1978-1988.

15. Tamura H, Fukada M, Fujikawa A, Noda M: Protein tyrosine phosphatase receptor type $\mathrm{Z}$ is involved in hippocampus-dependent memory formation through dephosphorylation at Y1105 on p190 RhoGAP. Neurosci Lett 2006, 399(1-2):33-38.

16. Pariser H, Perez-Pinera P, Ezquerra L, Herradon G, Deuel TF: Pleiotrophin stimulates tyrosine phosphorylation of beta-adducin through inactivation of the transmembrane receptor protein tyrosine phosphatase beta/zeta. Biochem Biophys Res Commun 2005, 335(1):232-239.

17. Pariser H, Ezquerra L, Herradon G, Perez-Pinera P, Deuel TF: Fyn is a downstream target of the pleiotrophin/receptor protein tyrosine phosphatase beta/zeta-signaling pathway: regulation of tyrosine phosphorylation of Fyn by pleiotrophin. Biochem Biophys Res Commun 2005, 332(3):664-669.

18. Meng K, Rodriguez-Pena A, Dimitrov T, Chen W, Yamin M, Noda M, Deuel TF: Pleiotrophin signals increased tyrosine phosphorylation of beta beta-catenin through inactivation of the intrinsic catalytic activity of the receptor-type protein tyrosine phosphatase beta/zeta. Proc Natl Acad SCi U S A 2000, 97(6):2603-2608.

19. Wang V, Davis DA, Veeranna RP, Haque M, Yarchoan R: Characterization of the activation of protein tyrosine phosphatase, receptor-type, $Z$ polypeptide 1 (PTPRZ1) by hypoxia inducible factor-2 alpha. PLoS One 2010, 5(3):e9641.

20. Ulbricht U, Eckerich C, Fillbrandt R, Westphal M, Lamszus K: RNA interference targeting protein tyrosine phosphatase zeta/receptor-type 
protein tyrosine phosphatase beta suppresses glioblastoma growth in vitro and in vivo. J Neurochem 2006, 98(5):1497-1506.

21. Foehr ED, Lorente G, Kuo J, Ram R, Nikolich K, Urfer R: Targeting of the receptor protein tyrosine phosphatase beta with a monoclonal antibody delays tumor growth in a glioblastoma model. Cancer Res 2006, 66(4):2271-2278

22. Ulbricht U, Brockmann MA, Aigner A, Eckerich C, Muller S, Fillbrandt R, Westphal M, Lamszus K: Expression and function of the receptor protein tyrosine phosphatase zeta and its ligand pleiotrophin in human astrocytomas. J Neuropathol Exp Neurol 2003, 62(12):1265-1275.

23. Muller S, Kunkel P, Lamszus K, Ulbricht U, Lorente GA, Nelson AM, von Schack D, Chin DJ, Lohr SC, Westphal M, et al: A role for receptor tyrosine phosphatase zeta in glioma cell migration. Oncogene 2003, 22(43):6661-6668.

24. Feng ZJ, Gao SB, Wu Y, Xu XF, Hua X, Jin GH: Lung cancer cell migration is regulated via repressing growth factor PTN/RPTP beta/zeta signaling by menin. Oncogene 2010, 29(39):5416-5426.

25. Simms E, Gazdar AF, Abrams PG, Minna JD: Growth of human small cell (oat cell) carcinoma of the lung in serum-free growth factor-supplemented medium. Cancer Res 1980, 40(12):4356-4363.

26. Carney DN, Gazdar AF, Bepler G, Guccion JG, Marangos PJ, Moody TW, Zweig MH, Minna JD: Establishment and identification of small cell lung cancer cell lines having classic and variant features. Cancer Res 1985, 45(6):2913-2923.

27. Nitadori J, Ishii G, Tsuta K, Yokose T, Murata Y, Kodama T, Nagai K, Kato H, Ochiai A: Immunohistochemical differential diagnosis between large cell neuroendocrine carcinoma and small cell carcinoma by tissue microarray analysis with a large antibody panel. Am J Clin Pathol 2006 125(5):682-692.

28. Ma Y, Ye F, Xie X, Zhou C, Lu W: Significance of PTPRZ1 and CIN85 expression in cervical carcinoma. Arch Gynecol Obstet 2011, 284(3):699-704.

29. Makinoshima H, Dezawa M: Pancreatic cancer cells activate CCL5 expression in mesenchymal stromal cells through the insulin-like growth factor-I pathway. FEBS Lett 2009, 583(22):3697-3703.

30. Chow JP, Fujikawa A, Shimizu H, Suzuki R, Noda M: Metalloproteinase- and gamma-secretase-mediated cleavage of protein-tyrosine phosphatase receptor type Z. J Biol Chem 2008, 283(45):30879-30889.

31. Barr AJ, Ugochukwu E, Lee WH, King ON, Filippakopoulos P, Alfano I, Savitsky P, Burgess-Brown NA, Muller S, Knapp S: Large-scale structural analysis of the classical human protein tyrosine phosphatome. Cell 2009, 136(2):352-363.

32. Blanchetot C, Tertoolen LG, Overvoorde J, den Hertog J: Intra- and intermolecular interactions between intracellular domains of receptor protein-tyrosine phosphatases. J Biol Chem 2002, 277(49):47263-47269.

33. Liang L, Lim KL, Seow KT, Ng CH, Pallen CJ: Calmodulin binds to and inhibits the activity of the membrane distal catalytic domain of receptor protein-tyrosine phosphatase alpha. J Biol Chem 2000, 275(39):30075-30081.

34. Benaim G, Villalobo A: Phosphorylation of calmodulin. Functional implications. Eur J Biochem 2002, 269(15):3619-3631.

35. Perez-Pinera P, Zhang W, Chang Y, Vega JA, Deuel TF: Anaplastic lymphoma kinase is activated through the pleiotrophin/receptor protein-tyrosine phosphatase beta/zeta signaling pathway: an alternative mechanism of receptor tyrosine kinase activation. $J$ Biol Chem 2007, 282(39):28683-28690

36. Corti C, Leclerc L'Hostis E, Quadroni M, Schmid H, Durussel I, Cox J, Dainese Hatt P, James P, Carafoli E: Tyrosine phosphorylation modulates the interaction of calmodulin with its target proteins. Eur J Biochem 1999, 262(3):790-802

37. Williams EL, Djamgoz MB: Nitric oxide and metastatic cell behaviour. Bioessays 2005, 27(12):1228-1238.

38. Fukumura D, Kashiwagi $S$, Jain RK: The role of nitric oxide in tumour progression. Nat Rev Cancer 2006, 6(7):521-534.
39. Eyler CE, Wu Q, Yan K, Macswords JM, Chandler-Militello D, Misuraca KL, Lathia JD, Forrester MT, Lee J, Stamler JS, et al: Glioma stem cell proliferation and tumor growth are promoted by nitric oxide synthase-2. Cell 2011, 146(1):53-66.

40. Jeong HC, Kim Gl, Cho SH, Lee KH, Ko JJ, Yang JH, Chung KH: Proteomic analysis of human small cell lung cancer tissues: up-regulation of coactosin-like protein-1. J Proteome Res 2011, 10(1):269-276.

doi:10.1186/1471-2407-12-537

Cite this article as: Makinoshima et al.: PTPRZ1 regulates calmodulin phosphorylation and tumor progression in small-cell lung carcinoma. BMC Cancer 2012 12:537.

\section{Submit your next manuscript to BioMed Central and take full advantage of:}

- Convenient online submission

- Thorough peer review

- No space constraints or color figure charges

- Immediate publication on acceptance

- Inclusion in PubMed, CAS, Scopus and Google Scholar

- Research which is freely available for redistribution

Submit your manuscript at www.biomedcentral.com/submit
( Biomed Central 